\title{
Very Special Relativity
}

\author{
Andrew G. Coher* and Sheldon L. Glashow \\ Physics Department, Boston University \\ Boston, MA 02215, USA
}

(Dated: Jan 26, 2006)

\begin{abstract}
By Very Special Relativity (VSR) we mean descriptions of nature whose space-time symmetries are certain proper subgroups of the Poincaré group. These subgroups contain space-time translations together with at least a 2-parameter subgroup of the Lorentz group isomorphic to that generated by $K_{x}+J_{y}$ and $K_{y}-J_{x}$. We find that VSR implies special relativity (SR) in the context of local quantum field theory or of $C P$ conservation. Absent both of these added hypotheses, VSR provides a simulacrum of SR for which most of the consequences of Lorentz invariance remain wholly or essentially intact, and for which many sensitive searches for departures from Lorentz invariance must fail. Several feasible experiments are discussed for which Lorentz-violating effects in VSR may be detectable.
\end{abstract}

Special relativity (SR) is based on the hypothesis that the laws of physics share many of the symmetries of Maxwell's equations. Whereas the maximal symmetry group of Maxwell's equations is the 15-parameter conformal group $S U(2,4)$, the existence of particles with mass (and the known violations of $P$ and $T$ ) constrains spacetime symmetry to be no greater than the Poincaré group (the connected component of the Lorentz group along with space-time translations). The special theory of relativity identifies this group as the symmetry of nature.

Although no decisive departure from exact Lorentz invariance has yet been detected, ever more sensitive searches should be and are being carried out. A perturbative framework has been developed to investigate a certain class of departures from Lorentz invariance. For example, Coleman and Glashow [1, 2] consider the case of space-time translations along with exact rotational symmetry in the rest frame of the cosmic background radiation, but allow small departures from boost invariance in this frame. Perturbative departures from Lorentzinvariance are then readily parametrized in terms of a fixed time-like 4-vector or 'spurion.' Others [3, 4] consider the introduction into the Lagrangian of more general spurion-mediated perturbations (sometimes referred to as 'expectation values of Lorentz tensors following spontaneous Lorentz breaking.').

In this note we pursue a different approach to the possible failure of Lorentz symmetry. We ask whether the exact symmetry group of nature may be isomorphic to a proper subgroup of the Poincaré group. To preserve energy and momentum conservation, we consider only those subgroups that include space-time translations along with a proper subgroup of the Lorentz group. Up to isomorphism and possible discrete elements, there are four such distinct subgroups with 1-parameter, three with 2-parameters, five with 3-parameters, and just one with 4-parameters.

For reasons soon to be explained, we restrict our attention to those subgroups of the Lorentz group containing the generators $T_{1} \equiv K_{x}+J_{y}$ and $T_{2}=K_{y}-J_{x}$, where
$\mathbf{J}$ and $\mathbf{K}$ are the generators of rotations and boosts respectively. These commuting generators form a group, $T(2)$, which is isomorphic to the group of translations in the plane. Three larger subgroups of the Lorentz group are obtained by adjoining one or two generators to $T(2)$. Each of them has a natural action on the plane: the addition of $J_{z}$ yields a group isomorphic to the threeparameter group of Euclidean motions, $E(2)$; the addition of $K_{z}$ yields one isomorphic to the three-parameter group of orientation-preserving similarity transformations, or homotheties, $\operatorname{HOM}(2)$; and lastly, the addition of both $J_{z}$ and $K_{z}$ yields one isomorphic to the fourparameter similitude group, $\operatorname{SIM}(2)[\underline{7}]$.

We refer to any scheme whose space-time symmetries consist of translations along with any one of the Lorentz subgroups described above as Very Special Relativity (VSR). We shall see that the four VSR avatars thus defined have quite different character. Nevertheless, they all share the following remarkable defining property: that the incorporation of either $P, T$ or $C P$ enlarges these subgroups to the full Lorentz group. Conjugation by one of these discrete transformations treats boosts and rotations oppositely, thereby extending the group to allow boosts and rotations in the $x-y$ plane independently. Further commutation leads to the remaining $z$-boost and $z$-rotation. The group $T(2)$ is the smallest subgroup of the Lorentz group with this property, the only others being those containing $T(2)$, hence our focus. It follows that Lorentz-violating effects in VSR are absent for theories conserving any one of these three discrete symmetries (and perhaps, that Lorentz-violating effects in VSR are necessarily small because $C P$ violating effects are small.)

In previous approaches, the breaking of Lorentz symmetry was expressed in terms of local operators incorporating one or more invariant tensors, or spurions. In the case of $S O(3)$ symmetry, the spurion takes the form of a time-like 4-vector, and the lowest dimension operators involving it affect both particle propagation and the kinematics of particle decays. The limits on such departures from SR in this model are exceptionally strong. For ex- 
ample, the mere observation of ultra-high energy cosmic rays places an upper bound of $10^{-23}$ on one dimensionless measure of Lorentz violation [1, while an analysis of neutrino data bounds flavor-dependent Lorentz violation in the neutrino sector to less than $10^{-25}[\underline{5}$ ].

We may attempt to apply a similar spurion strategy to the four VSR groups described above. The smallest group $T(2)$ admits many possible invariant tensors. The simplest of these whose little group is no greater than $T(2)$ is the antisymmetric two-index tensor [ $[8]$

$$
F=\left(\begin{array}{cccc}
0 & 1 & 0 & 0 \\
-1 & 0 & 0 & -1 \\
0 & 0 & 0 & 0 \\
0 & 1 & 0 & 0
\end{array}\right)
$$

( $F$ may be thought of as the field-strength for a zero frequency electromagnetic wave with linear polarization in the $x$-direction.)

The group $E(2)$ admits the 4 -vector $n=(1,0,0,1)$ as an invariant tensor. (This is also an invariant tensor for $T(2)$, but one which preserves rotations about the $z$-axis as well.) The existence of invariant tensors for the VSR groups $T(2)$ and $E(2)$ allows the construction of Lorentzviolating local operators that, among other things, affect the propagation of particles, much as in the $O(3)$ case. However unlike that case, these new operators necessarily violate $P, C P$ and $T$.

The remaining two VSR groups $H O M(2)$ and $S I M(2)$ are entirely different in this regard. There are no invariant tensors for these cases $\underline{9}$. No local Lorentz symmetry-breaking operator preserving either of these groups exists and there is no obvious local, perturbative description of their departures from SR. Consequently, spurions cannot access scenarios in which the symmetry group of nature is $\operatorname{HOM}(2)$ or $S I M(2)$.

The situation for these groups is much like that of $C P T$ in the context of Lorentz-invariant local quantum field theory: all local operators preserving Lorentz invariance preserve a larger symmetry (Lorentz plus $C P T$ ). Here, all local operators preserving $\operatorname{SIM}(2)$ (or $H O M(2)$ ) also preserve a larger symmetry (Lorentz). Nevertheless it is easy to construct non-local amplitudes that violate Lorentz invariance while respecting $S I M(2)$ (or $H O M(2))$.

One way to do this makes use of the non-invariant null vector $n \equiv(1,0,0,1)$. This vector is invariant under $T_{1}, T_{2}$ transformations and $z$-axis rotations, but transforms as $n \rightarrow e^{\phi} n$ under boosts in the $z$-direction. Consequently, ratios of dot-products of this vector with kinematic variables (such as momenta) are invariant under $S I M(2)$ or $H O M(2)$ but not under all Lorentz transformations. For example, the amplitude for the two body decay of a spinless particle at rest can depend on the 4 -momenta of the decay products, $p_{1}$ and $p_{2}$. The ratio $\left(p_{1} \cdot n\right) /\left(p_{2} \cdot n\right)$ is then an invariant, and thus the amplitude for the decay may depend on the direction of the decay products relative to the VSR-preferred direction (nominally, the $z$ axis).

Because VSR includes space-time translations, particle states may be labeled by their 4-momenta. For $S I M(2)$ and $\operatorname{HOM}(2)$, the only invariant that can be constructed from the 4-momentum of a massive particle is the mass itself, just as in SR. Therefore, all positive energy time-like momenta of fixed length are equivalent under $S I M(2)$ or $H O M(2)$ transformations. (A given time-like momentum may be transformed to the rest frame by three successive transformations: a $T_{1}$ transformation with parameter $-p_{x} /\left(E-p_{z}\right)$; a $T_{2}$ transformation with parameter $-p_{y} /\left(E-p_{z}\right)$; and a boost in the $z$-direction with parameter $e^{\phi}=\left(E-p_{z}\right) / M$.) This result implies that many of the elementary consequences of SR, such as timedilation, the law of velocity addition, the existence of a center-of-mass frame, and a universal and isotropic maximal attainable velocity hold in these variants of VSR. Indeed, invariance under $\operatorname{HOM}(2)$, rather than (as is often taught) the Lorentz group, is both necessary and sufficient to ensure that the speed of light is the same for all observers, and inter alia, to explain the null result to the Michelson-Morley experiment and its more sensitive successors.

Nature seems well described by the Standard Model, a Lorentz-invariant local quantum field theory in which the existence of three fermion families is necessary for $C P$ violation. As a result, $C P$ violating effects are usually small and are nearly absent in all flavor-diagonal processes. In this context, we note that the failure to detect the neutron electric dipole moment shows that $\bar{\theta}<10^{-10}$, while arguments suggest that $\bar{\theta}$ may be considerably smaller.

Were $C P$ an exact symmetry, VSR would imply SR. Consequently, because $C P$ violating effects in nature are small, we expect VSR departures from SR to be correspondingly small. However, such departures may be a dominant effect in processes for which $C P$ violation is significant. For example, in the decay $K_{L} \rightarrow \pi^{+}+\pi^{-}$, the pions need not be isotropically distributed in the kaon rest frame. Their directions could be correlated to the VSR-preferred direction. This effect is likely to be tiny for the spurion-inaccessible variants of VSR, because $C P$ violation in kaon decay is predominantly indirect (propagator dominated) and as we have noted, particle propagation is unaffected for $\operatorname{SIM}(2)$ or $\operatorname{HOM}(2)$.

For the spurion-inaccessible variants of VSR, observable departures from SR might be found in studies of the $C P$-violating decays of neutral $B$ mesons (such as $B_{0} \rightarrow J / \Psi+K_{s}$ ), where $C P$ violation is largely direct and significant angular correlations may be present. A straightforward search for departures from SR can be performed without knowing the times of individual events, so long as the VSR preferred direction $\vec{n}$ is not coincident with the Earth's polar axis. In that case, we anticipate an angular distribution (in the $B$ rest frame) 
depending on the angle between a decay product and the polar axis.

VSR may have radical consequences for neutrino physics. Neutrinos are now known to have mass. Several mechanisms have been contrived to remedy the absence of neutrino mass in the Standard Model. All of these invoke new particles or new interactions. In the 'Dirac' picture, lepton number is conserved with neutrinos acquiring mass via (anomalously small) Yukawa couplings to sterile $S U(2)$-singlet neutrinos. In the 'Majorana' picture, lepton number is violated. Neutrino masses result from a seesaw mechanism involving heavy sterile states, or via dimension- 6 operators resulting from unspecified new interactions.

In VSR, neutrino mass has a natural origin. Leptonnumber conserving neutrino masses, although not Lorentz invariant, are VSR invariant. There is no guarantee that neutrino masses have a VSR origin, but if so their sizes may be an indication of the magnitude of Lorentz-violating effects in other sectors. For example VSR allows for an (anisotropic!) electric dipole moment for charged leptons. $S U(2)$ invariance may then relate such dipole moments to neutrino masses: $d_{\text {lepton }} \sim\left(m_{\nu} / 2 m_{l}\right)^{2}\left(e / 2 m_{l}\right)$. For the electron and for $m_{\nu}^{2} \simeq 10^{-4} \mathrm{eV}^{2}$ this is the same size as the current experimental sensitivity $[\underline{6}$. We leave detailed explication of these and related matters to a subsequent publication.

A VSR origin of neutrino masses requires no additional states and need not introduce lepton number violation [10]. This is a significant departure from conventional notions. However, because all observable neutrino phenomena involve ultra-relativistic neutrinos $(\gamma>>1)$, neutrino phenomenology is virtually identical to that of the usual scenarios: the neutrino helicity will differ significantly (but unobservably) from $-1 / 2$, but only in a narrow cone about the preferred axis with opening angle $\sim 1 / \gamma$; and neutrinoless double beta decay is forbidden (and therefore also unobservable) by lepton number conservation.

Previous authors 1, 2, 3, 4] have noted that spurionmediated Lorentz violation can lead to two varieties of potentially observable Lorentz-violating effects: $C P T$ conserving or $C P T$ violating. The same is true for VSR in its spurion-accessible variants, $T(2)$ and $E(2)$. However, this is not necessarily the case for VSR in its $S I M(2)$ avatar. The $C P T$ operation is equivalent to a complex VSR transformation: a rotation about the $z$-axis by $\pi$ along with an imaginary boost by the same amount in the $z$-direction. Thus for amplitudes satisfying appropriate analyticity properties, $C P T$ follows from $S I M(2)$. (This argument is similar to the canonical proof of $C P T$ invariance in Lorentz invariant theories). While complex $H O M(2)$ can reverse the sign of any given 4-vector, $C P T$ invariance is not implied because the necessary transformation is momentum-dependent.

Our paper initiates an exploration of the possibility that the many empirical successes of special relativity need not demand Lorentz invariance of the underlying theoretical framework. Could the Lorentz invariant Standard Model emerge as an effective theory from a more fundamental scheme, perhaps operative at the Planck scale, that is VSR (but not SR) invariant? Such a scheme, as we have noted, cannot be a precisely local quantum field theory and its effects, especially for the case of the spurion-inaccessible variants of VSR, are difficult to estimate.

AGC was supported in part by the Department of Energy under grant no. DE-FG02-01ER-40676; SLG by the National Science Foundation under grant no. nsf-phy 0099529

* Electronic address: cohen@bu.edu

$\dagger$ Electronic address: slg@bu.edu

[1] S. R. Coleman and S. L. Glashow, Phys. Rev. D59, 116008 (1999), hep-ph/9812418.

[2] S. R. Coleman and S. L. Glashow, Phys. Lett. B405, 249 (1997), hep-ph/9703240.

[3] D. Colladay and V. A. Kostelecky, Phys. Rev. D55, 6760 (1997), hep-ph/9703464.

[4] D. Colladay and V. A. Kostelecky, Phys. Rev. D58, 116002 (1998), hep-ph/9809521.

[5] G. Battistoni et al., Phys. Lett. B615, 14 (2005), hepex/0503015.

[6] B. C. Regan, E. D. Commins, C. J. Schmidt, and D. DeMille, Phys. Rev. Lett. 88, 071805 (2002).

[7] There is a further one-parameter family of threedimensional groups in which we adjoin the generator $K_{z}+a J_{z}$ for any real, non-zero $a$. We do not consider these here.

[8] For a finite-dimensional representation of the Lorentz group labeled by two non-negative half-integers $(n, m)$, such an invariant tensor corresponds to the state with weight $|n,-m\rangle$. To avoid invariance under the larger group $E(2) n$ must differ from $m$.

[9] For an irreducible representation labeled by two halfintegers $(n, m)$ the only state annihilated by $T_{1}$ and $T_{2}$ is $|n,-m\rangle$. But this is an eigenstate of $K_{z}$ with eigenvalue $n+m$ which vanishes only for $n=m=0$.

[10] The massless and massive unitary representations of $S I M(2)$ and $\operatorname{HOM}(2)$ are one-dimensional, unlike those of the Poincaré group. 